6-1986

\title{
Electron Detachment in Negative-Ion Collisions. III. Model Calculations.
}

\section{T. S. Wang}

John B. Delos

William \& Mary, jbdelos@wm.edu

Follow this and additional works at: https://scholarworks.wm.edu/aspubs

Part of the Physics Commons

\section{Recommended Citation}

Wang, T. S. and Delos, John B., Electron Detachment in Negative-Ion Collisions. III. Model Calculations. (1986). Physical Review A, 33(6), 3832-3841.

https://doi.org/10.1103/PhysRevA.33.3832

This Article is brought to you for free and open access by the Arts and Sciences at W\&M ScholarWorks. It has been accepted for inclusion in Arts \& Sciences Articles by an authorized administrator of W\&M ScholarWorks. For more information, please contact scholarworks@wm.edu. 


\title{
Electron detachment in negative-ion collisions. III. Model calculations
}

\author{
T. S. Wang and J. B. Delos \\ Physics Department, College of William and Mary, Williamsburg, Virginia 23185
}

(Received 16 October 1985)

\begin{abstract}
With the use of a previously developed close-coupling theory, and simple models for the energy gap and propagator that arise in that theory, calculations are made of the properties of the survival probability for the negative ions and of the energy spectrum of detached electrons. Special attention is given to interference effects that might be seen under favorable circumstances.
\end{abstract}

\section{INTRODUCTION}

A theory of electron detachment in collisions of negative ions with atoms has been developed in earlier papers. ${ }^{1}$ The purpose of the present paper is to explore some of the possible consequences of that theory. Making use of simplified models, we shall calculate and display some qualitative properties of the negative-ion survival probability and of the detached-electron energy spectrum.

The theory developed in Ref. 1 is based on a semiclassical close-coupling framework. In Ref. 1(d) we showed that by neglecting intracontinuum transitions, the timedependent coupled equations can be reduced to a single integro-differential equation, which contains two functions: the energy gap $\Delta(t)$ between ionic and neutral states, and a propagator $\mathscr{G}\left(t, t^{\prime}\right)$ which is related to the coupling between bound and free states. Hence an $a b$ initio calculation of electron detachment cross sections could be made by calculating these two functions, and then solving the integro-differential equation.

Energy gaps as functions of internuclear distance have been calculated for a number of systems, ${ }^{2}$ but an $a b$ initio calculation of the propagator would be a major undertaking. Moreover, several of the phenomena that appear in cross sections seem to be primarily related to the energy gap $\Delta(t)$, and they probably are not terribly sensitive to the detailed form of the propagator. For example, it appears that the isotope effect ${ }^{1(c), 3,4}$ in the total detachment cross section depends mainly on $\Delta(t)$ : if the discrete state crosses into the continuum, then (at low energies) the heavier isotope (e.g., $\mathrm{D}^{-}$) undergoes more detachment than the lighter one $\left(\mathrm{H}^{-}\right)$, whereas if there is no such crossing, then the opposite effect is found.

It follows that many interesting results can be obtained by using simplified models for $\mathscr{G}\left(t, t^{\prime}\right)$. In this paper we will particularly focus our attention on the energy spectrum of detached electrons. Under appropriate circumstances, this spectrum could show a number of effects that are directly related to the form of $\Delta(t)$.

The calculations reported here were partly motivated by the following qualitative considerations. Suppose we have a system for which the energies of ionic and neutral states cross in the manner indicated in Fig. 1(a). Then on a trajectory for which the turning point is $R_{1}$, the energy gap as a function of time has the form indicated on the left-
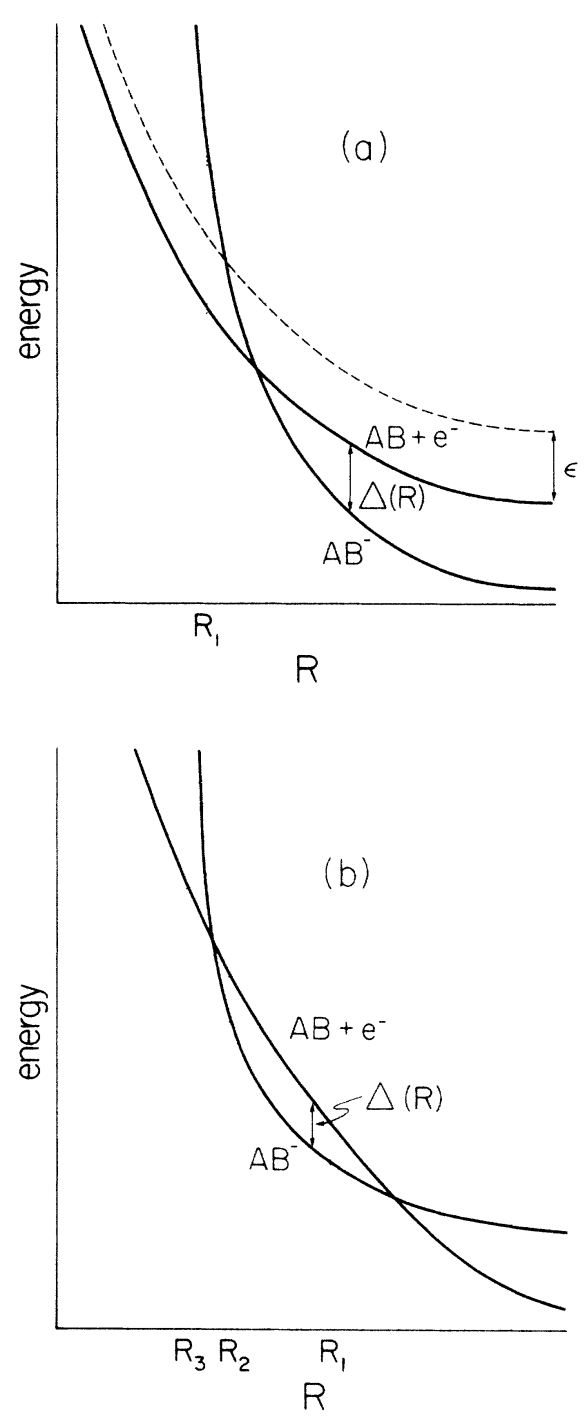

FIG. 1. (a) Schematic illustration of a curve crossing with a continuum. Solid lines are energies of the bound or ionic $\left(A B^{-}\right)$ and free or neutral $\left(A B+e^{-}\right)$states, where in the latter case the electron has zero kinetic energy. Dashed line is a free state in which the electron has kinetic energy $\epsilon . R_{1}$ is the turning point for a particular collision. (b) In some systems, the energy of the discrete state crosses into the continuum and then crosses back out at smaller $R$. 


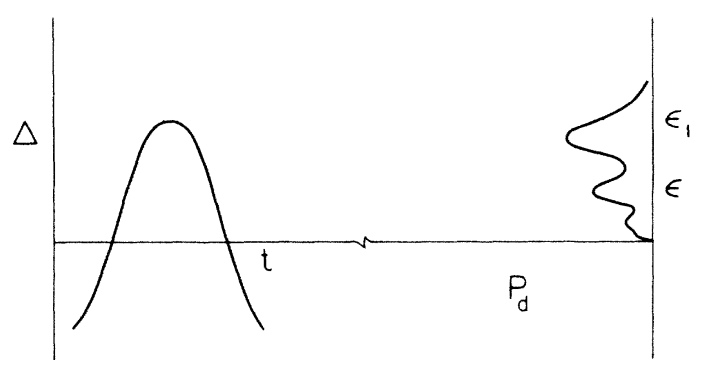

FIG. 2. For a trajectory with turning point $R_{1}$ in Fig. $1, \Delta(t)$ has the shape shown on the left. A corresponding hypothetical electron energy spectrum is shown on the right, as the detachment probability $P_{d}$ as a function of electron energy.

hand side of Fig. 2. The possible behavior of the resulting (doubly differential electron energy spectrum is indicated to the right: most electrons will have energies less than $\epsilon_{1}=\max \Delta(t)=\Delta(t=0)$, a type of "rainbow" may appear near $\epsilon_{1}$, there may be an interference pattern below $\epsilon_{1}$, and the behavior near $\epsilon=0$ should be consistent with the Wigner threshold law.

Such phenomena by themselves would be interesting enough, but even more complicated structures might occur. For the $\mathrm{H}^{-}-\mathrm{He}$ system (and perhaps for others as well), the energy of the discrete state crosses into the continuum, and then crosses back out again at smaller $R$, as indicated qualitatively in Fig. 1(b). For a trajectory with turning point $R_{1}$, the structure shown in Fig. 2 is again possible. For a trajectory with turning point $R_{2}$, the energy gap $\Delta(t)$ has the qualitative form indicated in Fig. 3. In this case there could be a rainbow at $\epsilon_{1}=\max \Delta(t)$, an inverted rainbow at $\epsilon_{2}=\Delta(t=0)$, a complicated four-term interference pattern between them, a simple two-term interference pattern below $\epsilon_{2}$, and again a Wigner-type threshold effect. The pattern would change with impact parameter in an interesting way. (The structure turns out to be related to a degenerate case of the swallowtail catastrophe. ${ }^{5}$ This will be shown in an appendix.)

While there is no doubt that such phenomena could exist (related phenomena are well known in Penning ioniza-

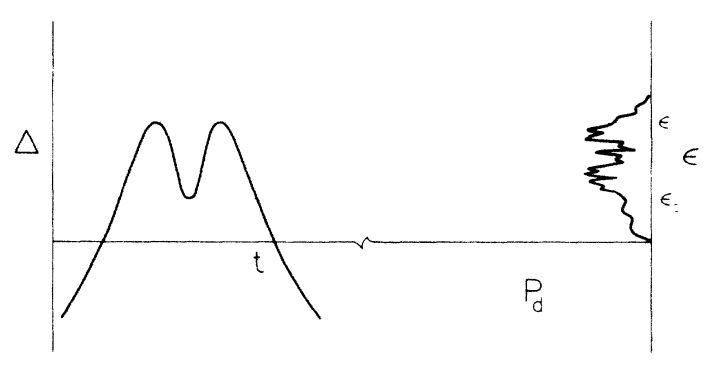

FIG. 3. For a trajectory with turning point $R_{2}$ in Fig. 1(b) $\Delta(t)$ has the shape shown on the left, and a hypothetical electron energy spectrum is shown on the right. tion $^{6}$ ), it is desirable to know whether or not they actually would be found in negative-ion collisions, and what their appearance might be. We present in this paper calculations of electron energy spectra based on simple approximations to $\Delta(t)$ and $\mathscr{G}\left(t, t^{\prime}\right)$. While we have not found anything as complicated as the hypothetical behavior indicated in Figs. 2 and 3, we show that oscillatory structure in the electron energy spectrum can in some cases be very clear.

In the following section, a brief summary of the theory is presented. In Sec. III we give the survival probability and electron energy spectrum assuming that the energy gap is a quadratic function of time; this is appropriate for systems with energy curves like those in Fig. 1(a). Subsequently, in Sec. IV, we carry out the same calculations for an energy gap that is a quartic function of time [cf. Fig. 1(b)].

\section{THEORY}

Calculations presented here are based on the closecoupling theory presented in Refs. $1(\mathrm{~d})$ and $1(\mathrm{e}) . C_{-1}(t)$ represents the probability amplitude for finding the electron in the bound state, and $C_{\epsilon}(t)$ is the amplitude for finding it in the free state with energy $\epsilon$. These amplitudes satisfy an infinite set of coupled differential equations. For solving these equations, we defined in Ref. 1(e) a "dynamical complex potential" $\mathscr{E}(t)$ such that

$$
C_{-1}(t)=\exp \left[-i \int_{t_{0}}^{t} \mathscr{E}\left(t^{\prime}\right) d t^{\prime} / \hbar\right)
$$

This equation (2.1) is the definition of $\mathscr{E}(t)$-in other words, this function is defined by this relationship to $C_{-1}(t)$, which is the exact solution to the close-coupled equations describing transitions between bound and free states. We have shown previously that under conditions discussed in Ref. $1(\mathrm{e}), \mathscr{E}(t)$ is approximately equal to the static complex potential that represents the shift and width of an unstable state in the continuum. However, in this paper we do not make use of that fact. Instead we calculate $\mathscr{E}(t)$ using the first-order approximation: ${ }^{1(\mathrm{e})}$

$\mathscr{E}(t)=\Delta(t)+\int_{0}^{\infty} \mathscr{G}(\tau ; t) \exp \left[i \int_{t-\tau}^{t} \Delta\left(t^{\prime}\right) d t^{\prime} / \hbar\right] d \tau$.

For the propagator $\mathscr{G}(\tau ; t)$ we use in this paper the "separable approximation" and "short-memory approximation" that were discussed in Ref. 1(d), together with the approximation $g(t)=1$, so

$$
\mathscr{G}(\tau ; t) \simeq \hat{\mathscr{G}}(\tau)
$$

and we take $\hat{Y}(\tau)$ to be the function plotted in Fig. 1 of Ref. 1(d).

With an assumed form for $\Delta(t), \mathscr{E}(t)$ can be evaluated using (2.2); then the survival amplitude is given in (2.1), and the final survival probability of the negative ion is

$$
\begin{aligned}
P_{s} & =\left|C_{-1}(t \rightarrow \infty)\right|^{2} \\
& =\exp \left[2 \operatorname{Im} \int_{-\infty}^{\infty} \mathscr{E}\left(t^{\prime}\right) d t^{\prime}\right] .
\end{aligned}
$$

[Probably a word needs to be said about the end points $\pm \infty$ here and below. Initial conditions are applied at $t_{0}$, 
long before the collision, and final amplitudes are examined at $t_{f}$, long after the collision; in principle we are supposed to take the limit $t_{0} \rightarrow-\infty, t_{f} \rightarrow \infty$. As was shown in Ref. 1(d), our phase conventions are such that the phases of $C_{-1}(t)$ and $C_{\epsilon}(t)$ do not have limits, but the magnitudes $\left|C_{-1}(t)\right|$ and $\left|C_{\epsilon}(t)\right|$ do possess definite limits. We write $\pm \infty$ as end points of integrals when the limit of required absolute value exists.]

To obtain the amplitude for detachment into the free state with energy $\epsilon$, we begin with Eqs. (2.13) of Ref. 1(d):

$$
C_{\epsilon}(t)=\exp \left(-i \Phi_{\epsilon}\left(t, t_{0}\right) / \hbar\right]\left[C_{\epsilon}\left(t_{0}\right)+(i \hbar)^{-1} \int_{t_{0}}^{t} d t^{\prime} V_{\epsilon,-1}\left(t^{\prime}\right) \exp \left[i \Phi_{\epsilon}\left(t^{\prime}, t_{0}\right) / \hbar\right] C_{-1}\left(t^{\prime}\right)\right]
$$

If the electron is initially in the bound state, then $C_{\epsilon}\left(t_{0}\right)=0$. Continuum states are defined such that their energies are independent of $R$, and therefore [Eq. (3.3) of Ref. 1(d)]

$$
\Phi_{\epsilon}\left(t_{1}, t_{2}\right)=\left(t_{1}-t_{2}\right) \epsilon .
$$

Hence it follows that

$$
C_{\epsilon}(t)=(i \hbar)^{-1} \int_{t_{0}}^{t} V_{\epsilon,-1} \exp \left[i \epsilon\left(t^{\prime}-t\right) / \hbar\right] C_{-1}\left(t^{\prime}\right) d t^{\prime}
$$

and the electron energy spectrum associated with a single trajectory is

$$
P_{d}(\epsilon)=\left|C_{\epsilon}(t \rightarrow \infty)\right|^{2} \rho_{\epsilon} .
$$

Consistent with the approximations made earlier for $\mathscr{G}$, we neglect the time dependence of $V_{\epsilon,-1}\left(t^{\prime}\right)$, and, combining (2.6), (2.5), and (2.1), we obtain

$$
P_{d}(\epsilon)=[\Gamma(\epsilon) / \hbar] \Pi^{2}(\epsilon)
$$

with

$$
\Gamma(\epsilon)=2 \pi\left|V_{-1, \epsilon}\right|^{2} \rho_{\epsilon}
$$

and

$$
\begin{aligned}
\Pi(\epsilon)= & (2 \pi \hbar)^{-1 / 2} \\
& \times\left|\int_{-\infty}^{\infty} \exp \left[-i \int_{-\infty}^{t}\left[\mathscr{E}\left(t^{\prime}\right)-\epsilon\right] d t^{\prime} / \hbar\right) d t\right| .
\end{aligned}
$$

This integral will be evaluated using stationary-phase and uniform approximations that will be discussed later.

In principle, the probabilities of survival and of detachment are supposed to add to unity,

$$
P_{s}+\int_{0}^{\infty} P_{d}(\epsilon) d \epsilon=1,
$$

but since $\mathscr{E}(t)$ is here calculated by an approximation (2.2), probability conservation is not guaranteed, so (2.10) provides a useful check on the overall accuracy of the results. In our calculations, we found that $(2.10)$ is satisfied within a few percent. ${ }^{7}$

\section{QUADRATIC APPROXIMATION TO $\Delta(t)$}

The energy gap $\Delta$ between the bound state and the continuum is a function of the internuclear distance $R$, and in the semiclassical picture, $R$ is a function of time with $d^{2} R / d t^{2}$ related to the average force between the colliding particles. If the time $t=0$ is chosen to correspond to the point of closest approach, then reasonable models of the average force lead to functions $R(t)$ which are symmetric in $t$. Hence $\Delta(t) \equiv \Delta(R(t))$ is a symmetric function of time, and the present calculations are based upon the simplest possible models for this function.

In this section we consider a quadratic approximation to $\Delta(t)$ :

$$
\Delta(t)=E_{0}-\beta t^{2},
$$

where $E_{0}$ and $\beta$ are constants. Such an approximation is suitable for describing situations like those indicated in Figs. 1(a) and 2. In such cases, for a small range of $R$ near the crossing point $R_{x}$ (or turning point $R_{\mathrm{TP}}$ ), $\Delta(R)$ is approximately linear in $R$,

$$
\Delta(R) \simeq\left(F_{\text {neutral }}-F_{\text {ion }}\right)\left(R-R_{x}\right),
$$

and the average acceleration is approximately constant,

$$
R \simeq R_{\mathrm{TP}}+(\bar{F} / \mu)\left(t^{2} / 2\right)
$$

so $\Delta(t)$ is approximately quadratic in $t$. We consider this approximation first because it is much simpler than the higher-degree approximation that is needed to describe the situation shown in Figs. 1(b) and 3, and because we want to compare our results with those given by Taylor and Delos, who solved the close-coupled equations by different methods.

Reasonable values for the parameters in this model are easily estimated. $E_{0}$ represents the maximum energy gap $\Delta_{\max }$ for a path having some specified impact parameter. For various paths in various systems, $E_{0}$ would typically range between -0.2 and $+2 \mathrm{eV}(\sim-0.01$ to +0.1 hartrees): for typical negative-ion systems, the maximum penetration of the discrete state into the continuum is a few $\mathrm{eV}$, and, on the other hand, if the energy gap is too large ( $E_{0}$ too negative) then detachment is very improbable. To estimate $\beta$, we note that

$$
2 \beta=-\left.\frac{d^{2} \Delta}{d t^{2}}\right|_{\mathrm{TP}}=-\frac{d \Delta}{d R} \frac{d^{2} R}{d t^{2}}=-\frac{d \Delta}{d R} \frac{\bar{F}}{\mu} .
$$

The average force $\bar{F}$ and $d \Delta / d R$ are typically a few eV per Bohr radius, and reduced masses of various systems would range between $10^{3}$ and $10^{5}$ electron mass units, so we find that $\beta$ would be between $10^{-5}$ and $10^{-7}$ atomic units.

\section{A. Survival probability}

For $\beta=10^{-6}$, we show in Fig. 4 a graph of the survival probability $P_{s}$ as a function of $E_{0}$, calculated by numerical integration of Eqs. (2.2) and (2.9). This result is compared with that of the classical local-complex-potential model, and with the result calculated by Taylor and Delos. ${ }^{1(b)}$ 


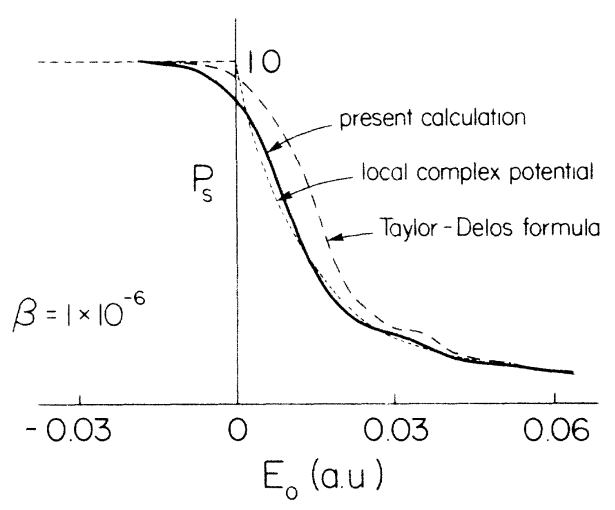

FIG. 4. Survival probability in the quadratic model as a function of the parameter $E_{0}$ for $\beta=10^{-6}$. Present results ( - ) are compared with those of Taylor and Delos (...-+ .) and with the classical local-complex-potential results ( $-\ldots)$.

ment, Taylor and Delos used most of the same assumptions that are used in the present development, and they solved the same close-coupled equations. Their methods were quite different, but in most respects, their approximations were analogous to those used here. The present calculation differs from that in Ref. 1(b) in one significant way: in Ref. 1(b), when the survival probability was calculated, a term that was third order in the coupling strength was neglected; in the present calculation, the dynamical complex potential $\mathscr{E}(t)$ was calculated using the first-order approximation discussed in Ref. 1(e) [the method used here is identical to that used to obtain Fig. 3 of Ref. 1(e)]. These two approximations should be of comparable accuracy, but there is not direct correspondence between them, so the results given in Ref. 1(b) need not be identical to those given here (also it is not possible to say which of the two more accurately represents the exact survival probability). In Fig. 5 we see that the present results are close to those given in Ref. 1(b).

The classical local-complex-potential results follow from our formulas if the slow-collision approximation is made. We showed in Figs. 1-4 of Ref. 1(e) that the dynamical complex potential oscillates about the local complex potential. The oscillations are not small, but when $\mathscr{E}(t)$ is integrated to obtain the survival probability, those oscillations have little effect and $P_{s}$ lies close to the "local" result. Small residual wiggles are visible in the solid curve in Fig. 4.

\section{B. Electron energy spectrum}

We now turn to the electron energy spectrum. Direct numerical computation of the multiple integrals that are implicit in Eq. (2.9) would be rather time consuming, so we shall simplify the problem further. We neglect the real part of the integral on the right-hand side of (2.2). This approximation has the same effect as does making a small $(\sim 10 \%)$ shift in $\Delta(t)$, and in view of the fact that our function $\Delta(t)$ is rather arbitrarily chosen, such a shift has no relevance. The integral (2.9) is then evaluated using a stationary-phase approximation and a uniform approximation.

\section{Stationary-phase approximation}

Since we have neglected the real part of $\mathscr{E}(t)-\Delta(t)$, the stationary-phase points of the integral (2.9) are the two roots of $\Delta(t)-\epsilon=0$, i.e.,

$$
\begin{aligned}
& t_{1}=-\left[\left(E_{0}-\epsilon\right) / \beta\right]^{1 / 2}, \\
& t_{2}=-t_{1} .
\end{aligned}
$$

We get different results depending upon whether $t_{1}$ and $t_{2}$ are real or imaginary.

a. $\epsilon<E_{0}$. For this classically accessible region, $t_{1}$ and $t_{2}$ are both real, and the stationary-phase (SP) approximation gives

$$
\left|C_{\epsilon}(\infty)\right|^{2}=\left(\pi V_{-1, \epsilon}^{2} / \beta t_{2}\right)\left(s_{1}^{2}+s_{2}^{2}+2 s_{1} s_{2} \sin \gamma\right),
$$

where

$$
\begin{aligned}
& s(t)=\exp \left(\int_{-\infty}^{t} \operatorname{Im} \mathscr{E}\left(t^{\prime}\right) d t^{\prime} / \hbar\right), \\
& s_{1}=s\left(t_{1}\right), \\
& s_{2}=s\left(t_{2}\right), \\
& \gamma=\frac{4}{3}\left(E_{0}-\epsilon\right)^{3 / 2} / \beta^{1 / 2} .
\end{aligned}
$$

From Eq. (3.7), $s^{2}(t)$ is equal to the survival probability at time $t$. We also know that $2 \pi V_{-1, \epsilon}^{2}$ is the detachment rate and that $2 \beta t_{2}$ is the rate of change of $\epsilon_{\mathrm{SP}}$ which we get from $\Delta(t)-\epsilon_{\mathrm{SP}}=0$. Hence we see from Eq. (3.6) that the first two terms of the right-hand side correspond to probabilities of detachment into the state with energy $\epsilon$ on the incoming and outgoing parts of the trajectory, at times $t_{1}$ and $t_{2}$ at which $\Delta\left(t_{i}\right)=\epsilon$. The third term corresponds to the interference between these two processes. [Compare Fig. 1 of Ref. 1(b).]

b. $\epsilon>E_{0}$. In this classically inaccessible region we have two stationary-phase points $t_{1}$ and $t_{2}$ which are both purely imaginary. From the condition that the integral must be convergent, we know that the contour of integration must be distorted so that it goes through the stationary-phase point $t_{2}$ in the lower half-plane. The value of the argument of the exponent at the stationaryphase point $t_{2}$ is

$$
\begin{aligned}
& (-i / \hbar) \int_{-\infty}^{t_{2}}\left[\mathscr{E}\left(t^{\prime}\right)-\epsilon\right] d t^{\prime} \\
& =(-i / \hbar)\left(\int_{-\infty}^{0}\left[\mathscr{E}\left(t^{\prime}\right)-\epsilon\right] d t^{\prime}\right. \\
& \left.\quad+\int_{0}^{t_{2}}\left[\mathscr{E}\left(t^{\prime}\right)-\epsilon\right] d t^{\prime}\right) .
\end{aligned}
$$

Since there is only one relevant stationary-phase point (so no interference effects in the forbidden region) and since we only need the absolute value of the integral in (2.9), we need only the real part of the integral above, and that is

$$
\int_{-\infty}^{0} \operatorname{Im} \mathscr{E}\left(t^{\prime}\right) d t^{\prime}+\int_{0}^{t_{2}}\left[\operatorname{Re} \mathscr{E}\left(t^{\prime}\right)-\epsilon\right]\left|d t^{\prime}\right| .
$$

We assume that the approximation $\operatorname{Re} \mathscr{E}\left(t^{\prime}\right) \sim \Delta\left(t^{\prime}\right)$ holds 
also on the imaginary axis for small $\left|t_{2}\right|$. It then follows that

$$
\left|\exp (-i / \hbar) \int_{-\infty}^{t_{2}}\left[\mathscr{E}\left(t^{\prime}\right)-\epsilon\right] d t^{\prime}\right|=s_{0} \exp \left(-\frac{1}{2}|\gamma|\right),
$$

where $s_{0}=s(t=0)$ and $\gamma$ are defined in Eqs. (3.7) and (3.8). Completing the integral using the stationary-phase method (actually it is the "steepest-descents" method in this case), one easily obtains

$$
\left|C_{\epsilon}(\infty)\right|^{2} \simeq\left|\pi V_{-1, \epsilon}^{2} / \beta t_{2}\right| s_{0}^{2} \exp (-|\gamma|) .
$$

Clearly $\left|C_{\epsilon}(\infty)\right|^{2}$ decreases exponentially in the forbidden region.

\section{Uniform approximation}

The stationary-phase approximation breaks down when the denominator $\beta t_{2}$ vanishes, i.e., when $\epsilon \sim E_{0}$, and for this region a uniform approximation is needed. All of the relevant formulas have been given by Conner and Marcus. $^{8}$ Let us define

$$
\begin{aligned}
& y=\left(E_{0}-\epsilon\right) / \beta^{1 / 2}, \\
& \hat{s}^{2}=s_{1} s_{2}, \\
& f_{1}=2 \beta t_{1}, \\
& f_{2}=2 \beta t_{2},
\end{aligned}
$$

where $t_{1}, t_{2}, s_{1}$, and $s_{2}$ are the same as in Eqs. (2.4), (2.5), (2.7), and (2.8).

We can express $\left|C_{\epsilon}(\infty)\right|^{2}$ as follows depending on whether $\epsilon$ is greater than or smaller than $E_{0}$.

a. $\epsilon<E_{0}$. In this classically accessible case, where $t_{1}$ and $t_{2}$ are real, we have

$$
\begin{aligned}
\left|C_{\epsilon}(\infty)\right|^{2}= & {\left[\pi^{2} V_{-1, \epsilon}^{2} / \beta t_{2} \hbar^{2}\right] } \\
& \times\left\{\left(s_{1}+s_{2}\right)^{2} y^{1 / 2} \mathrm{Ai}^{2}(-y)\right. \\
& \left.+\left(s_{1}-s_{2}\right)^{2} y^{-1 / 2}\left[\mathrm{Ai}^{\prime}(-y)\right]^{2}\right\},
\end{aligned}
$$

where $\mathrm{Ai}$ is the Airy function.

b. $\epsilon>E_{0}$. In this classically inaccessible region we have

$$
\begin{aligned}
& t_{1}=-i\left[\left(\epsilon-E_{0}\right) / \beta\right]^{1 / 2}, \\
& t_{2}=-t_{1},
\end{aligned}
$$

and

$$
\begin{aligned}
\left|C_{\epsilon}(\infty)\right|^{2} & \\
= & \frac{4 \pi^{2} \hat{s}^{2} V^{2}-1, \epsilon}{f_{0} \hbar^{2}} \\
& \times\left\{[1-\sin (\alpha-\pi / 2)]|y|^{1 / 2} \mathrm{Ai}(|y|)\right. \\
& \left.+[1+\sin (\alpha-\pi / 2)]|y|^{-1 / 2}\left[\mathrm{Ai}^{\prime}(|y|)\right]^{2}\right\},
\end{aligned}
$$

where

$$
\begin{aligned}
& \alpha=i \int_{t_{1}}^{t_{2}} \operatorname{Im} \mathscr{E}\left(t^{\prime}\right) d t^{\prime} / \hbar, \\
& f_{0}=\left[4 \beta\left(\epsilon-E_{0}\right)\right]^{-1 / 2} .
\end{aligned}
$$

There is a problem in applying Eq. (3.14). The quantity $\alpha$ in (3.15a) involves an integral over $\mathscr{E}(t)$ for purely imaginary $t$, but we have only calculated $\mathscr{E}(t)$ for real $t$. There are several ways of estimating $\alpha$, of which the simplest is the approximation $\alpha=0$. This can be justified in the following way. The classically inaccessible region starts where $\epsilon-E_{0}$ is zero, and both $t_{1}$ and $t_{2}$ are zero. As $\epsilon$ increases, $t_{1}$ and $t_{2}$ move out the imaginary axis and the magnitude of $\epsilon$ increases. However, when $\epsilon$ is large enough that $\alpha$ is significant, the Airy functions and $\left|C_{\epsilon}(\infty)\right|^{2}$ have become very small. In the range of $\epsilon$ in which $\left|C_{\epsilon}(\infty)\right|^{2}$ is significant, $\alpha$ is close to zero, and Eq. (3.14) can be simplified to
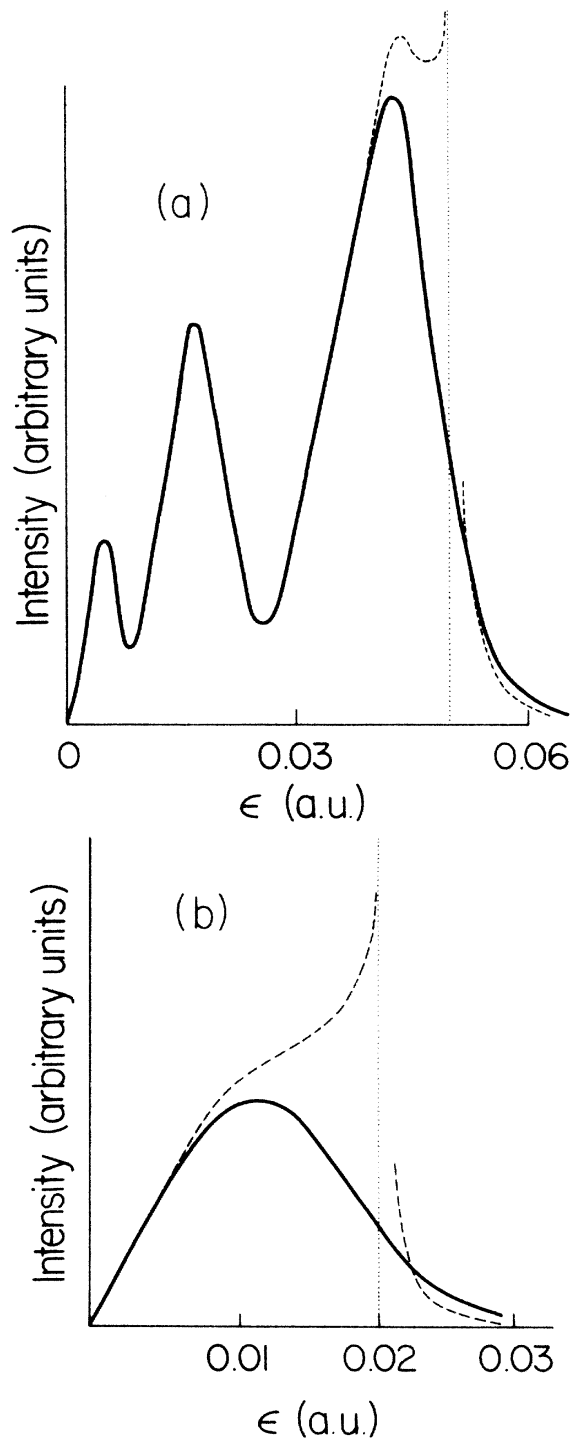

FIG. 5. (a) Doubly differential electron energy spectrum for $E_{0}=0.05$ and $\beta=10^{-6}$. (b) Same for $E_{0}=0.02$. 


$$
\left|C_{\epsilon}(\infty)\right|^{2}=\frac{8 \pi^{2} \hat{s}^{2} V_{-1, \epsilon}^{2}}{f_{0} \hbar^{2}}|y|^{1 / 2} \mathrm{Ai}^{2}(|y|)
$$

\section{Results}

In Figs. 5(a) and 5(b) we show the calculated $P_{d}(\epsilon)$ using stationary-phase and uniform approximations for $\beta=10^{-6}$ and $E_{0}=+0.05$ and 0.02 , respectively. Interference oscillations are quite evident in the first case, but they do not appear when $E_{0}$ is too small, as would be expected from the formulas and interpretation given earlier.

\section{QUARTIC APPROXIMATION TO $\Delta(t)$}

In the preceding section, we used a quadratic approximation to $\Delta(t)$. As explained in the Introduction, such an approximation is relevant to situations shown in Figs. 1(a) and 2. To describe the more complicated situation shown in Figs. 1(b) and 3, a more elaborate form for $\Delta(t)$ is needed. The simplest approximation that has the appropriate behavior when $\Delta(t) \geq 0$ is

$$
\Delta(t)=E_{0}+\beta t^{2}-\alpha t^{4} .
$$

For $\alpha, \beta$ both positive, this $\Delta(t)$ has a maximum of

$$
\epsilon_{1}=\Delta_{\max }=E_{0}+\beta^{2} / 4 \alpha
$$

when

$$
t= \pm(\beta / 2 \alpha)^{1 / 2}
$$

and a local minimum value of $\epsilon_{2}=E_{0}$ at $t=0$. Arguments similar to those in the preceding section tell us that typical values for $\alpha$ would be around $10^{-10}$ atomic units.

\section{A. Dynamical complex potential and survival probability}

For the case $E_{0}=0.03, \beta=1 \times 10^{-5}, \alpha=5 \times 10^{-10}$, we show in Fig. 6 the functions $\Delta(t), \hat{G}(\Delta(t))$, and $\mathscr{E}(t)$ $-\Delta(t)$, which oscillates about $\widehat{G}(\Delta(t))$.

Figure 7 shows the survival probability $P_{s}$ as a function of $E_{0}$ for various combinations of $\alpha$ and $\beta$. The effective threshold for electron detachment occurs when the discrete curve just touches the continuum, i.e., at $E_{0}=-\beta^{2} / 4 \alpha$. This contrasts with the quadratic case, for which the effective threshold is at $E_{0}=0$. Vague hints of interference structure appear in some of the curves, but generally such effects cannot be very significant in the survival probability.

\section{B. Electron energy spectrum}

As in Sec. II B, stationary-phase and uniform approximations can be used to calculate the electron energy spec-
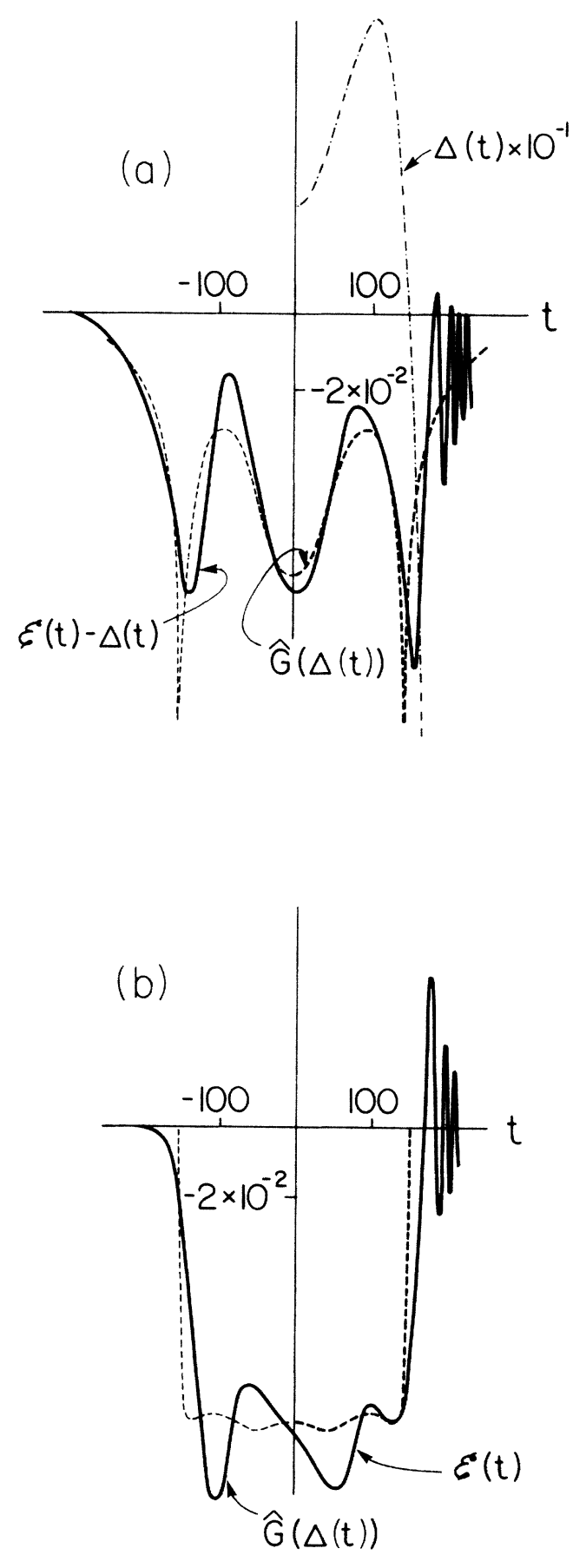

FIG. 6. (a) Real part of static and dynamical complex potentials: The energy gap $\Delta(t)$ is reduced by a factor of 10 to display it on this scale. Dashed line is the real part of $\hat{G}(\Delta(t))$, which in a local-complex-potential theory represents the energy shift of the unstable state. The real part of the dynamical complex potential $\mathscr{E}(t)-\Delta(t)$ follows $\hat{G}(\Delta(t))$ rather closely, but lacks cusps, and has rapid oscillations for large positive $t$. (b) Imaginary part of static and dynamic complex potentials. The static complex potential represents the width of the unstable state. Again the dynamical complex potential is close to the static one but is smoother near the crossing point, and more oscillatory elsewhere. 


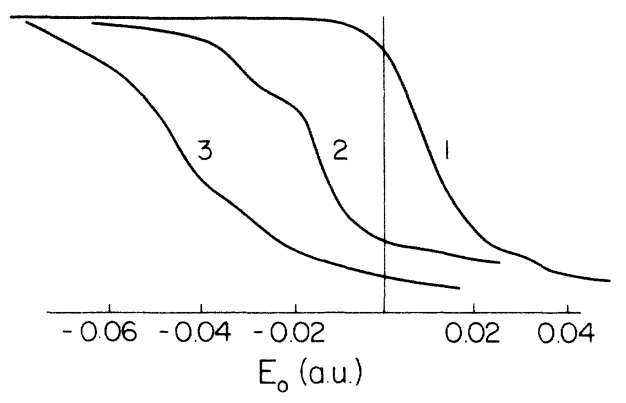

FIG. 7. Survival probability in the quartic model.

trum for this model. Since the formulas are rather more complicated here than they are for the quadratic case, we put them in an appendix, and only give the results and explanation.

Figure 8(a) shows the electron energy spectrum for $\beta=10^{-5}, \alpha=5 \times 10^{-10}$, and $E_{0}=+0.03$. In this case, $\Delta(t)$ has a shape like that in Fig. 3, and this would correspond to a turning point near $R_{2}$ in Fig. $1(\mathrm{~b})$. Figures $8(\mathrm{~b})$ and $8(\mathrm{c})$ show the spectrum for $E_{0}=0.0$ and -0.03 , respectively; these would correspond to turning points near $R_{3}$ and $R_{4}$ in Fig. 3.

In the stationary-phase approximation, there are four real or complex stationary-phase points: referring again to Fig. 3, if $0<\epsilon<\epsilon_{2}$, then two are real and two are complex; if $\epsilon_{2}<\epsilon<\epsilon_{1}$, all four are real, and if $\epsilon_{1}<\epsilon$ all four are complex. The result is a complicated combination of oscillatory and exponential terms which can lead to the interesting structure shown in the figures.

\section{SUMMARY AND CONCLUSION}

Using very simple models for the energy gap and continuum propagator, we have calculated the survival probability and electron energy spectrum for electrons detaching from negative ions. We have shown that under favorable circumstances the electron energy spectrum could show interesting interference structure. Such effects are well known for Penning ionization, and the present calculations indicate that they may sometimes also arise in negative-ion collisions.

However, our calculations also indicate that interference effects in collisional detachment will rarely be as dramatic as they are in the case of Penning ionization. There are two reasons for this. First, the phase of the oscillations is determined by $\int \Delta\left(t^{\prime}\right) d t^{\prime} / \hbar$, the integral over time of the energy gap between discrete and continuum states. For systems undergoing Penning ionization the energy gap can be some tens of $\mathrm{eV}$, while for negative-ion systems this gap is rarely more than 1 or $2 \mathrm{eV}$. Hence only in quite slow collisions will $\int \Delta d t / \hbar$ become large enough to produce dramatic oscillations. Second, the magnitude of the oscillations is small unless $\int \Gamma\left(t^{\prime}\right) d t^{\prime} / \hbar$ is not too large. Interference arises from multiple paths leading to the same final state; if the negative molecular ion produced temporarily in the collision is very unstable with respect to electron detachment, then detachment takes place with high probability as soon as the bound
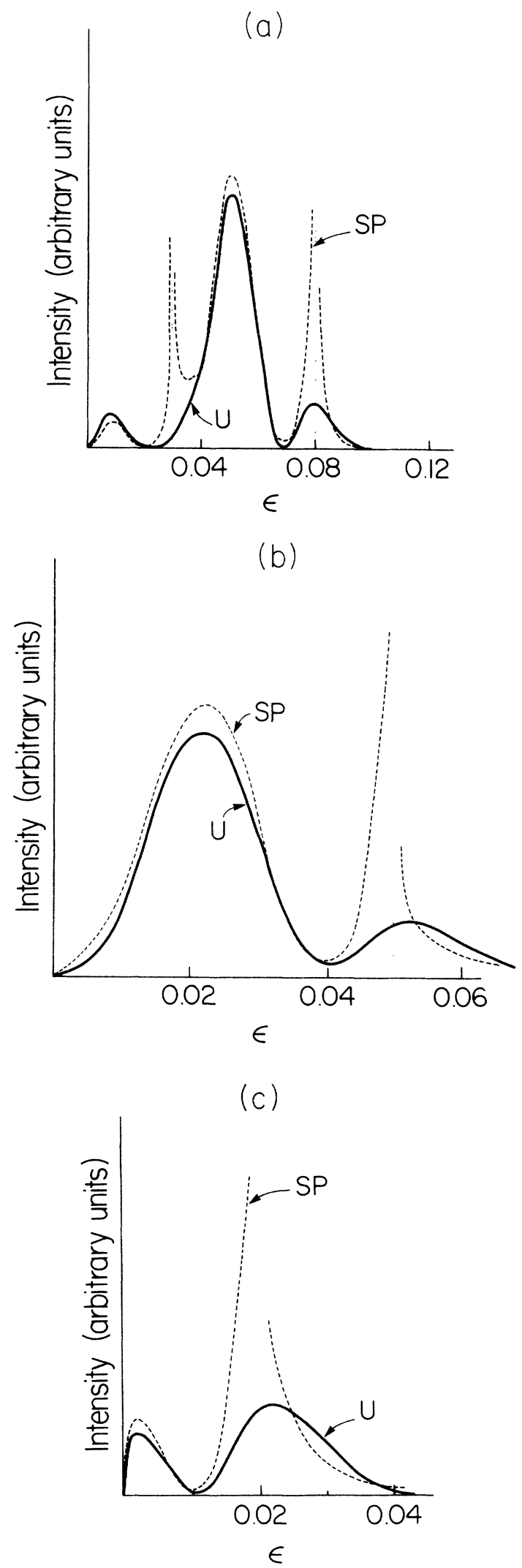

FIG 8. (a) Electron energy spectrum in the quartic model for $E_{0}=+0.03, \beta=10^{-5}, \alpha=5 \times 10^{-10}$. Dashed line is the stationary-phase approximation, and solid line is the uniform approximation. $\epsilon$ is given in a.u. (b) Same for $E_{0}=0$. (c) Same for $E_{0}=-0.03$. 
state crosses over into the continuum, and few negative ions survive to produce interference effects. Little quantitative information is available about the lifetimes of these states, but it is known that they are normally very short.

For $\mathrm{H}^{-}$-rare-gas systems, measurements, previous calculations using other theories, ${ }^{9}$ and calculations using close-coupling theory which we shall report in a future publication generally show no oscillations (through in a few cases these are little bumps that might be caused by interference). This is not surprising, since energy gaps in those cases are always very small.

One system for which such interference might appear in doubly differential (and possibly singly differential) measurements is $\mathrm{Na}^{-}$on $\mathrm{H}$. According to the calculations of Olson and $\mathrm{Liu}$, the $\mathrm{Na}^{-}-\mathrm{H}$ energy crosses into the continuum at an internuclear separation of about $8 a_{0}$, then remains about $0.5 \mathrm{eV}$ above the continuum for an extended range. At $10 \mathrm{eV}$ (c.m.) collision energy, the phase $\int \Delta\left(t^{\prime}\right) d t^{\prime} / \hbar$ would be on the order of $3 \pi$, certainly large enough to produce oscillations. Avoided crossings which appear among the nearby discrete states embedded in the continuum should also produce interesting effects. Of course it is not known whether the lifetimes of these states are long enough for any such effects to be visible.

\section{ACKNOWLEDGMENT}

This work was supported by the National Science Foundation.

\section{APPENDIX: FORMULAS FOR THE ELECTRON ENERGY SPECTRUM FOR THE QUARTIC CASE}

\section{Stationary-phase approximation}

Defining

$$
f(t)=\frac{1}{5} \alpha t^{5}-\frac{1}{3} \beta t^{3}-\left(E_{0}-\epsilon\right) t
$$

the stationary-phase points are the roots of $d f(t) / d t \equiv \Delta(t)-\epsilon=0$, from which we have

$$
\begin{aligned}
& t_{1}=\left(\left\{\beta+\left[\beta^{2}+4 \alpha\left(E_{0}-\epsilon\right)\right]^{1 / 2}\right\} / 2 \alpha\right)^{1 / 2}, \\
& t_{2}=-t_{1}, \\
& t_{3}=\left(\left\{\beta-\left[\beta^{2}+4 \alpha\left(E_{0}-\epsilon\right)\right]^{1 / 2}\right\} / 2 \alpha\right)^{1 / 2}, \\
& t_{4}=-t_{3} .
\end{aligned}
$$

Three cases arise (Fig. 3). If $\epsilon>\epsilon_{1}=\max _{t} \Delta(t)=E_{0}$ $+\beta^{2} / 4 \alpha$, there are four complex stationary-phase points. If $0<\epsilon<\epsilon_{2}=\Delta(t=0)=E_{0}$, there are two real and two complex stationary-phase points. Finally, if $\epsilon_{2}<\epsilon<\epsilon_{1}$, there are four real stationary-phase points.

$$
\text { a. } E_{0}<\epsilon<E_{0}+\beta^{2} / 4 \alpha
$$

We have throughout this region

$$
\left.\left|C_{\epsilon}(\infty)\right|=\left.\left|\sum_{k=1}^{4}\right| \frac{2 \pi}{\dot{\Delta}\left(t_{k}\right)}\right|^{1 / 2} V_{-1, \epsilon} s\left(t_{k}\right) \exp \mid-i \frac{\pi}{4} \operatorname{sgn} \dot{\Delta}\left(t_{k}\right)\right) \exp \int_{-\infty}^{t_{k}}(-i)[\Delta(t)-\epsilon] d t^{\prime} / \hbar \mid,
$$

where

$$
s\left(t_{k}\right)=\exp \left(\int_{-\infty}^{t_{k}} \operatorname{Im} \mathscr{E}(t) d t / \hbar\right) .
$$

$$
\text { b. } 0<\epsilon<E_{0}
$$

If $\epsilon<E_{0}, t_{3}$ and $t_{4}$ are purely imaginary, and the contour must be distorted so that it passes through $t_{1}$ and $t_{2}$ on the real axis and $t_{4}$ in the lower half-plane. The detachment amplitude $C_{\epsilon}$ is then a combination of three terms:

$$
\begin{aligned}
\left|C_{\epsilon}\right|^{2}=V_{-1, \epsilon}^{2} & \left.\left|\sum_{k=1}^{2}\right| \frac{2 \pi}{\dot{\Delta}\left(t_{k}\right)}\right|^{1 / 2} s\left(t_{k}\right) \exp \left[-i \frac{\pi}{4} \operatorname{sgn} \dot{\Delta}\left(t_{k}\right)\right] \exp (i / \hbar) \int_{-\infty}^{t_{k}}\left[\epsilon-\Delta\left(t^{\prime}\right)\right] d t^{\prime} \\
& +\left.\left|\frac{2 \pi}{\dot{\Delta}\left(t_{4}\right)}\right|^{1 / 2} s(0) \exp \left[\frac{i}{\hbar} \int_{-\infty}^{t_{4}}\left[\epsilon-\Delta\left(t^{\prime}\right)\right] d t^{\prime}\right]\right|^{2} .
\end{aligned}
$$

$$
\text { c. } \epsilon>E_{0}+\beta^{2} / 4 \alpha
$$

For this classically inaccessible region we have

$$
\begin{aligned}
& t_{1}=a_{0}+i b_{0}, \\
& t_{2}=-t_{1}, \\
& t_{3}=t_{1}^{*}, \\
& t_{4}=-t_{1}^{*},
\end{aligned}
$$

where

$$
\begin{aligned}
& a_{0}=\left[\frac{\beta}{4 \alpha}+\left[\frac{\epsilon-E_{0}}{4 \alpha}\right]^{1 / 2}\right]^{1 / 2}, \\
& b_{0}=\left[-\frac{\beta}{4 \alpha}+\left[\frac{\epsilon-E_{0}}{4 \alpha}\right]^{1 / 2}\right]^{1 / 2} .
\end{aligned}
$$

The stationary-phase points are all complex, and the contour of integration must be distorted so that it passes 
through the two that lie in the lower half-plane; those are $t_{2}$ and $t_{3}$. Then the stationary-phase approximation gives

$$
\begin{aligned}
\left|C_{\epsilon}(\infty)\right|=\left.\left|\sum_{k=2}^{3}\right| \frac{2 \pi}{\dot{\Delta}\left(t_{k}\right)}\right|^{1 / 2} V_{-1, \epsilon} s\left(\operatorname{Re}\left(t_{k}\right)\right) \\
\left.\qquad \exp \mid(-i / \hbar) \int_{-\infty}^{t_{k}}\left[\Delta\left(t^{\prime}\right)-\epsilon\right] d t^{\prime}\right) \mid,
\end{aligned}
$$

where the sum is over two terms, with $k=2$ and $k=3$. Here we have again used the same approximation that was used in going from (3.9a) to (3.9c): that $\operatorname{Re} \mathscr{E}(t) \simeq \Delta(t)$ holds in a narrow strip of the complex plane.

Finally, let us note that the stationary-phase approximation breaks down in regions where $\epsilon$ is close to $E_{0}$ or to $E_{0}+\beta^{2} / 4 \alpha$, where $d \Delta(t) / d t=0$.

\section{Uniform approximation}

Connor ${ }^{8}$ has developed general methods for the approximate evaluation of integrals similar to the ones appearing here.

We first put the integral into his standard form by transforming to a new variable

$$
\begin{aligned}
& c_{0}=(\alpha / 5)^{-1 / 5}, \\
& x=t / c_{0}, \\
& \zeta_{1}=\left(\epsilon-E_{0}\right) c_{0}, \\
& \zeta_{3}=(-\beta / 3) c_{0}^{3}, \\
& f(x)=\zeta_{1} x+\zeta_{3} x^{3}+x^{5}, \\
& U\left(\zeta_{1}, \zeta_{3}\right)=\int_{-\infty}^{\infty} \exp [i f(x)] d x .
\end{aligned}
$$

Here and below we use atomic units so $\hbar=1$. Then

$$
\left|C_{\epsilon}(\infty)\right|=V_{\epsilon,-1} c_{0}\left|\int_{-\infty}^{\infty} s(t(x)) \exp [i f(x)] d x\right| \text {. }
$$

Next $s(t(x))$ is expanded in a four-term Taylor series in $x$,

$$
c_{0} s(t(x))=q_{0}+q_{1} x+q_{2} x^{2}+q_{3} x^{3} .
$$

(The rule is that the number of terms in this expansion should be equal to the number of stationary-phase points.) The coefficients $q_{0}, \ldots, q_{3}$ are evaluated by the requirement that

$$
\begin{aligned}
c_{0} s_{k} \equiv c_{0} s\left(t_{k}\right) & \equiv c_{0} s\left(t_{k}\left(x_{k}\right)\right) \\
& =q_{0}+q_{1} x_{k}+q_{2} x_{k}^{2}+q_{3} x_{k}^{3},
\end{aligned}
$$

where $t_{k}$ and $x_{k}$ are the stationary-phase points. This gives four simultaneous linear equations, which are to be solved for $q_{k}$ 's.

Finally, noting that for any integer $p$,

$$
\begin{aligned}
& \int x^{p} \exp [i f(x)] d x=\left(-i \partial / \partial \zeta_{1}\right)^{p} U\left(\zeta_{1}, \zeta_{3}\right), \\
& \int x^{3 p} \exp [i f(x)] d x=\left(-i \partial / \partial \zeta_{3}\right)^{p} U\left(\zeta_{1}, \zeta_{3}\right),
\end{aligned}
$$

we can insert (A9) into (A8) to obtain

$$
\begin{aligned}
\left|C_{\epsilon}(\infty)\right|=V_{-1, \epsilon} & {\left[q_{0} U-q_{2} \frac{\partial^{2} U}{\partial \xi_{1}^{2}}\right.} \\
& \left.-i\left[q_{1} \frac{\partial U}{\partial \xi_{1}}+q_{3} \frac{\partial U}{\partial \xi_{3}}\right)\right] .
\end{aligned}
$$

The function $U$ and its derivatives can be expressed in terms of gamma functions:

$$
\begin{aligned}
& U\left(\zeta_{1}, \zeta_{3}\right)=2 \sum_{l_{1}=0}^{\infty} \sum_{l_{3}=0}^{\infty}\left(\xi_{1}^{l_{1}} / l_{1} !\right)\left(\xi_{3}^{l_{3}} / l_{3} !\right) F\left(l_{1}, l_{3}\right), \\
& \frac{\partial U}{\partial \xi_{1}}=2 \sum_{l_{1}=1}^{\infty} \sum_{l_{3}=0}^{\infty}\left[\xi_{1}^{l_{1}-1} /\left(l_{1}-1\right) !\right]\left(\xi_{3}^{l_{3}} / l_{3} !\right) F\left(l_{1}, l_{3}\right), \\
& \frac{\partial^{2} U}{\partial \xi_{1}^{2}}=2 \sum_{l_{1}=2}^{\infty} \sum_{l_{3}=0}^{\infty}\left[\xi_{1}^{l_{1}-2} /\left(l_{1}-2\right) !\right]\left(\xi_{3}^{l_{3}} / l_{3} !\right) F\left(l_{1}, l_{3}\right), \\
& \frac{\partial U}{\partial \xi_{3}}=2 \sum_{l_{1}=0}^{\infty} \sum_{l_{3}=1}^{\infty}\left(\xi_{1}^{l_{1}} / l_{1} !\right)\left[\xi_{3}^{l_{3}-1} /\left(l_{3}-1\right) !\right] F\left(l_{1}, l_{3}\right),
\end{aligned}
$$

where

$$
\begin{aligned}
F\left(l_{1}, l_{3}\right)= & \Gamma\left(\frac{1}{5}\left(1+l_{1}+3 l_{3}\right)\right) \\
& \times \cos \left[\frac{\pi}{10}\left(1+6 l_{1}+8 l_{3}\right)\right] .
\end{aligned}
$$

The formulas for $\left(q_{0}, q_{1}, q_{2}, q_{3}\right)$ differ according to whether $\epsilon$ is in classically accessible or inaccessible regions.

$$
\text { a. } \epsilon<E_{0}+\beta^{2} / 4 \alpha
$$

In this classically accessible region we solve the linear equations to obtain for the $q$ 's,

$q_{0}=\left(c_{0} / 2\right)\left[x_{3}^{2}\left(s_{1}+s_{2}\right)-x_{1}^{2}\left(s_{3}+s_{4}\right)\right] /\left(x_{3}^{2}-x_{1}^{2}\right)$,

$q_{1}=\left(c_{0} / 2\right)\left[x_{3}^{2}\left(s_{1}-s_{2}\right)-x_{1}^{2}\left(s_{3}-s_{4}\right)\right] / x_{1} x_{3}\left(x_{3}^{2}-x_{1}^{2}\right)$,

$q_{2}=\left(c_{0} / 2\right)\left(s_{1}+s_{2}+s_{3}+s_{4}\right) /\left(x_{1}^{2}-x_{3}^{2}\right)$,

$q_{3}=\left(c_{0} / 2\right)\left[x_{3}\left(s_{1}-s_{2}\right)-x_{1}\left(s_{3}-s_{4}\right)\right] / x_{1} x_{3}\left(x_{3}^{2}-x_{1}^{2}\right)$,

where, as stated earlier

$$
\begin{aligned}
& x_{k}=c_{0} t_{k}, \\
& s_{k}=\exp \left(c_{0} \int_{-\infty}^{x_{k}} \operatorname{Im} \mathscr{E}\left(c_{0} x\right) d x\right) .
\end{aligned}
$$

and $t_{k}$ 's are given in Eq. (A2).

In the case of $\epsilon<E_{0}$, we make the approximation that $x_{3}=x_{4}=0$.

$$
\text { b. } \epsilon>E_{0}+\beta^{2} / 4 \alpha
$$

For this classically inaccessible case, Eqs. (A13) still hold, but it is better to separate real and imaginary parts of $t_{k}$ using (A4). In these equations we can also neglect the imaginary parts of $t_{k}$ 's when calculating $s_{k}$ 's. It follows that $s_{1}=s_{3}$ and $s_{2}=s_{4}$, and the formulas simplify to 


$$
\begin{aligned}
& q_{0}=c_{0}\left(s_{1}+s_{2}\right) / 2, \\
& q_{1}=c_{0}\left(s_{1}-s_{2}\right)\left(3 a_{0}^{2}-b_{0}^{2}\right) / 4 a_{0}^{2}\left(a_{0}^{2}+b_{0}^{2}\right), \\
& q_{2}=0, \\
& q_{3}=-c_{0}\left(s_{1}-s_{2}\right) / 4 a_{0}^{2}\left(a_{0}^{2}+b_{0}^{2}\right) .
\end{aligned}
$$

Equations (A13) or (A15) together with (A10)-(A12) give the results shown in Fig. 8.

\section{Connection with catastrophe theory}

Enthusiasts of catastrophe theory will recognize Eq. (A1) as a special case of the universal unfolding of the swallowtail catastrophe. With $t$ as the "internal" or "state" variable, the swallowtail involves the "germ" $t^{5}$, and the "universal unfolding"

$$
t^{5}+a t^{3}+b t^{2}+c t
$$

In the present case, because of the symmetry of $\Delta(t)$, the quadratic term $b t^{2}$ vanishes. It follows that the structure corresponds to a particular "cut' or "slice" of the swallowtail catastrophe.

The fact that our quartic model is connected with one of the canonical catastrophes establishes rigorously an intuitively obvious fact: the results we show here are "typical" or "generic"- they are not radically changed by small changes in the function $\Delta(t)$. 1(a) R. D. Taylor and J. B. Delos, Proc. R. Soc. London Ser. A 379, 179 (1982); (b) R. D. Taylor and J. B. Delos, Proc. R. Soc. London Ser. A 379, 209 (1982); (c) T. S. Wang and J. B. Delos, J. Chem. Phys. 79, 4306 (1983); (d) T. S. Wang and J. B. Delos, Phys. Rev. A 29, 542 (1984); (e) T. S. Wang and J. B. Delos, Phys. Rev. A 29, 552 (1984). Of these references, only (d) and (e) are essential for the present paper. (Correction: In Eqs. (4.3) and (5.2) of Ref. (e) $\exp \left[i(\pi / 4) \operatorname{sgn} \Delta\left(t_{x}^{n}\right)\right]$ should be $\exp \left[-i(\pi / 4) \operatorname{sgn} \Delta\left(t_{x}^{n}\right)\right]$. Also, the lower limit of the integral in Eq. (5.2) should be $t_{n}^{x}$.)

${ }^{2}$ R. E. Olson and B. Liu, Phys. Rev. A 17, 1568 (1978); 22, 1389 (1980); J. Chem. Phys. 73, 2817 (1980).

${ }^{3}$ R. L. Champion, L. D. Doverspike, and S. K. Lam, Phys. Rev. A 13, 617 (1976).

4J. P. Gauyacq, J. Phys. B 13, L501 (1980).

${ }^{5}$ T. I. Poston and I. Stewart, Catastrophe Theory and its Applica- tions (Pitman, London, 1978); J. N. L. Connor, Mol. Phys. 31, 33 (1976); 27, 853 (1974).

${ }^{6}$ W. H. Miller, J. Chem. Phys. 52, 3563 (1970); J. Lorenzen, H. Morgner, W. Bussart, M.-W. Ruf, and H. Hotop, Z. Phys. A 310, 141 (1983).

${ }^{7}$ Probability conservation is discussed in more detail by $\mathbf{M}$. $\mathbf{L}$. Du and J. B. Delos, Phys. Rev. A 33, 2294 (1986).

8J. N. L. Connor, Mol. Phys. 26, 1217 (1973); 26, 1371 (1973); 27, 853 (1974); J. N. L. Connor and R. A. Marcus, J. Chem. Phys. 55, 5636 (1971); J. N. L. Connor, P. R. Curtis, and D. Farrelly, J. Phys. A 17, 283 (1984).

${ }^{9}$ V. A. Esaulov, J. P. Grouard, R. I. Hall, M. Landau, J. L. Montmagnon, F. Pichou, and C. Schermann, J. Phys. B 17, 1855 (1984); V. N. Tuan, J. P. Gauyacq, and V. A. Esaulov, ibid. 16, L95 (1983). 